\title{
Constraining Dark Matter Models with a Light Mediator at the PandaX-II Experiment
}

Xiangxiang Ren, ${ }^{1}$ Li Zhao, ${ }^{1}$ Abdusalam Abdukerim, ${ }^{2}$ Xun Chen, ${ }^{1}$ Yunhua Chen, ${ }^{3}$ Xiangyi Cui, ${ }^{1}$ Deqing Fang, ${ }^{4}$ Changbo Fu, ${ }^{1}$ Karl Giboni, ${ }^{1}$ Franco Giuliani, ${ }^{1}$ Linhui Gu, ${ }^{1}$ Xuyuan Guo, ${ }^{3}$ Ke Han, ${ }^{1}$ Changda He, ${ }^{1}$ Di Huang, ${ }^{1}$ Shengming He, ${ }^{3}$ Xingtao Huang, ${ }^{5}$ Zhou Huang, ${ }_{1}^{1}$ Xiangdong Ji, ${ }^{1,6,7, *}$ Yonglin $\mathrm{Ju}^{8}{ }^{8}$ Yao Li, ${ }^{1}$ Heng Lin, ${ }^{1}$ Huaxuan Liu, ${ }^{8}$ Jianglai Liu, ${ }^{1,7}$ Yugang Ma, ${ }^{4}$ Yajun Mao, ${ }^{9}$ Kaixiang Ni, ${ }^{1}$ Jinhua Ning, ${ }^{3}$ Andi Tan, ${ }^{6}$ Hongwei Wang, ${ }^{4}$ Meng Wang, ${ }^{5}$ Qiuhong Wang, ${ }^{4}$ Siguang Wang, ${ }^{9}$ Xiuli Wang, ${ }^{8}$ Shiyong Wu, ${ }^{3}$ Jingkai Xia, ${ }^{1}$ Mengjiao Xiao, ${ }^{6,10}$ Pengwei Xie, ${ }^{1}$ Binbin Yan, ${ }^{5}$ Jijun Yang, ${ }^{1}$ Yong Yang, ${ }^{1, \dagger}$ Hai-Bo Yu, ${ }^{11,}$ Jianfeng Yue, ${ }^{3}$ Tao Zhang, ${ }^{1}$ Jifang Zhou, ${ }^{3}$ Ning Zhou, ${ }^{1}$ Qibin Zheng, ${ }^{12}$ and Xiaopeng Zhou ${ }^{9}$

(PandaX-II Collaboration)

\author{
${ }^{1}$ INPAC and School of Physics and Astronomy, Shanghai Jiao Tong University, \\ Shanghai Laboratory for Particle Physics and Cosmology, Shanghai 200240, China \\ ${ }^{2}$ School of Physics and Technology, Xinjiang University, Ürümqi 830046, China \\ ${ }^{3}$ Yalong River Hydropower Development Company, Ltd., 288 Shuanglin Road, Chengdu 610051, China \\ ${ }^{4}$ Shanghai Institute of Applied Physics, Chinese Academy of Sciences, Shanghai 201800, China \\ ${ }^{5}$ School of Physics and Key Laboratory of Particle Physics and Particle Irradiation (MOE), Shandong University, Jinan 250100, China \\ ${ }^{6}$ Department of Physics, University of Maryland, College Park, Maryland 20742, USA \\ ${ }^{7}$ Tsung-Dao Lee Institute, Shanghai 200240, China \\ ${ }^{8}$ School of Mechanical Engineering, Shanghai Jiao Tong University, Shanghai 200240, China \\ ${ }^{9}$ School of Physics, Peking University, Beijing 100871, China \\ ${ }^{10}$ Center of High Energy Physics, Peking University, Beijing 100871, China \\ ${ }^{11}$ Department of Physics and Astronomy, University of California, Riverside, California 92521, USA \\ ${ }^{12}$ School of Medical Instrument and Food Engineering, University of Shanghai for Science and Technology, Shanghai 200093, China
}

(Received 20 February 2018; revised manuscript received 28 May 2018; published 12 July 2018)

\begin{abstract}
We search for nuclear recoil signals of dark matter models with a light mediator in PandaX-II, a direct detection experiment in the China Jinping underground laboratory. Using data collected in 2016 and 2017 runs, corresponding to a total exposure of 54 ton day, we set upper limits on the zero-momentum dark matter-nucleon cross section. These limits have a strong dependence on the mediator mass when it is comparable to or below the typical momentum transfer. We apply our results to constrain self-interacting dark matter models with a light mediator mixing with standard model particles, and set strong limits on the model parameter space for the dark matter mass ranging from $5 \mathrm{GeV}$ to $10 \mathrm{TeV}$.
\end{abstract}

DOI: 10.1103/PhysRevLett.121.021304

The existence of dark matter (DM) is supported by a wide range of observations in astronomy and cosmology, but its particle nature remains elusive. Leading candidates such as weakly interacting massive particles (WIMPs) [1] that could explain the observed cosmological DM abundance, have been actively searched for in indirect and direct detection experiments, as well as at the Large Hadron Collider. The direct WIMP searches often assume a pointlike contact interaction between the DM candidate and the

Published by the American Physical Society under the terms of the Creative Commons Attribution 4.0 International license. Further distribution of this work must maintain attribution to the author(s) and the published article's title, journal citation, and DOI. Funded by SCOAP . nucleus, since the momentum transfer in nuclear recoils is much smaller than the weak-scale mediator mass. However, this assumption breaks down if the DM-nucleus interaction is mediated by a force carrier that has a mass comparable to or lighter than the momentum transfer [2-7].

Dark matter models with a light mediator are well motivated. For example, in many hidden-sector DM models [8-12], DM particles annihilate to the light mediator to achieve the observed abundance. It can induce an attractive potential between two DM particles and boost the annihilation cross section $[13,14]$. Furthermore, it has been shown the self-interacting DM (SIDM) model with a light mediator can explain observed stellar kinematics from dwarf galaxies to galaxy clusters $[15,16]$, a challenge for the prevailing cold DM model (see, e.g., Ref. [17]). If it couples to the standard model (SM) particles, the DM 
signal event in direct detection can be enhanced towards low recoil energies, a smoking-gun signature of SIDM $[4,6,7,18]$. In recent years, there has been great progress in the search for the light force mediator at the highluminosity facilities (see, e.g., Ref. [19] for a review).

In this Letter, we report upper limits on the DM-nucleon scattering cross section induced by a light mediator and then interpret them to constrain the SIDM models proposed in Ref. [4]. Our analysis is based on data from the PandaX-II experiment, which is the phase-II experiment in the PandaX project that consists of a series of xenonbased rare-event detection experiments, located at the China Jinping underground laboratory (CJPL). The central apparatus of PandaX-II is a dual-phase xenon time projection chamber (TPC). The active volume contains $580 \mathrm{~kg}$ liquid xenon. Particles interacting with xenon results in prompt scintillation photons ( $S 1$ signal) in liquid xenon as well as delayed electroluminescence photons ( $S 2$ signal) in gaseous xenon. Both signals are detected in one event by two arrays of photomultiplier tubes (PMTs), located in the top and bottom of the TPC. More detailed descriptions of the PandaX-II experiment can be found in Refs. [20-22].

We first consider a general case, where DM interacts with the nucleon through a vector or scalar force mediator, $\phi$, and further assume $\phi$ has equal effective couplings to the proton and neutron as in the standard WIMP model. The general form of the DM-nucleus elastic scattering cross section can be parametrized as [4]

$$
\sigma\left(q^{2}\right)_{\chi N}=\left.\sigma\right|_{q^{2}=0} A^{2}\left(\frac{\mu}{\mu_{p}}\right)^{2} \frac{m_{\phi}^{4}}{\left(m_{\phi}^{2}+q^{2}\right)^{2}} F^{2}\left(q^{2}\right),
$$

where $\left.\sigma\right|_{q^{2}=0}$ is the DM-nucleon cross section in the limit of zero momentum transfer $\left(q^{2}=0\right), A$ the mass number of the nucleus, $\mu\left(\mu_{p}\right)$ the DM-nucleus (nucleon) reduced mass, $m_{\phi}$ the mediator mass, and $F\left(q^{2}\right)$ the nuclear form factor. We see that $\sigma_{\chi N}$ is momentum dependent and it approaches the standard WIMP case when $m_{\phi} \gg q$.

The differential recoil rate (in unit of counts per day per $\mathrm{kg}$ per $\mathrm{keV}$ ) is [23]

$$
\frac{d R}{d E}=\frac{\sigma\left(q^{2}\right)_{\chi N} \rho}{2 m_{\chi} \mu^{2}} \int_{v \geq v_{\min }} d^{3} v v f(v, t),
$$

where $\rho$ is the local DM density which we set to be $0.3 \mathrm{GeV} / \mathrm{cm}^{3}, m_{\chi}$ is the DM particle mass, $f(v, t)$ is the time-dependent DM velocity distribution relative to the detector, and $v_{\min }$ is the minimum DM velocity that results in a recoil energy $E$.

This analysis uses the same data sets as the recent WIMP search (unblind) in PandaX-II [22], consisting of 80 live days of exposure in 2016 and 77 live days of exposure in 2017, the largest published data set of its kind to date. We apply the same event selection criteria as in Ref. [22]. The range for $S 1$ and $S 2$ signals are between 3 photoelectron (PE) and $45 \mathrm{PE}$, and $100 \mathrm{PE}$ (uncorrected) and $10000 \mathrm{PE}$, respectively. The total data were divided into 18 sets to take into account variations of detector parameters and background rates. Background contributions have been estimated, and no excess of events in data was observed above the background. For a given DM model, the expected event distributions are modeled with the same procedure as in Ref. [22]. For each data set, we simulate the expected $S 1$ and $S 2$ signal distributions from the SIDM recoil-energy spectra using a tuned NEST simulation framework. Then, we apply the experimental efficiencies to make further corrections.

Figure 1 shows the simulated $S 1$ distributions in PandaXII for a $100 \mathrm{GeV}$ DM particle with $m_{\phi}=10 \mathrm{MeV}$ (red) and a WIMP with the same mass (blue). Both cases have the same integrated rate, but their spectra are very different; i.e., the $S 1$ distribution of the light-mediator model is more peaked towards small $S 1$ than predicted in the WIMP model. We also plot PandaX-II detection efficiency as a function of $S 1$ (magenta). It is nearly a constant over the range of $10-45 \mathrm{PE}$, but is reduced dramatically for $S 1<10 \mathrm{PE}$, where the event rate of the light-mediator model is maximized. Thus, we expect DM direct detection sensitivity becomes weak when the mediator mass is comparable or less than the typical momentum transfer in nuclear recoils, even though the DM mass is still at the weak scale.

The same statistical method as in Ref. [22] is used to derive upper limits on the signal cross section. An unbinned

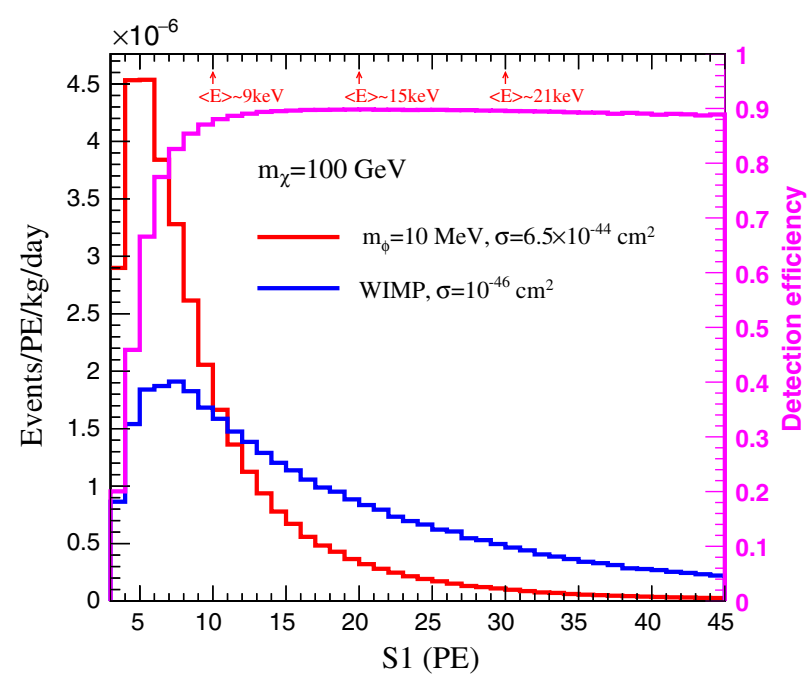

FIG. 1. Simulated $S 1$ spectrum (red) in PandaX-II for $\left(m_{\chi}, m_{\phi}\right)=(100,0.01) \mathrm{GeV}$ with the zero-momentum DMnucleon cross section fixed to be $6.5 \times 10^{-44} \mathrm{~cm}^{2}$. The distribution (blue) for the same DM mass but with a contact interaction is also shown. We take the WIMP-nucleon cross section to be $10^{-46} \mathrm{~cm}^{2}$ so both spectra have the same integrated rate. The detection efficiency vs $S 1$ (magenta) is plotted with the corresponding value labeled on the right vertical axis. The rough conversion between $S 1$ and recoil energy is indicated near the top horizontal axis for $S 1=10,20$, and $30 \mathrm{PE}$. 
likelihood function is constructed for these 18 data sets using the signal and background probability density functions in the $S 1-\log _{10}(S 2 / S 1)$ plane, taking into account the normalization uncertainties for signal and background. For the DM signal, we assign a conservative $20 \%$ uncertainty, estimated from different NEST simulations and uncertainties on the detector parameters. The standard profile likelihood ratio test statistic [24,25] is evaluated at grids of expected signal cross section (hypotheses) and compared to the test statistic distribution obtained from a large number of toy Monte Carlo data produced and fitted using the same signal hypotheses.

Figure 2 shows the 90\% confidence level (C.L.) upper limits on the zero-momentum DM-nucleon cross section assuming a light mediator with mass ranging from $1 \mathrm{MeV}$ to $1 \mathrm{GeV}$. In the left panel, we plot the limits as a function of the mediator mass for three representative DM masses, $10 \mathrm{GeV}$, $100 \mathrm{GeV}$, and $1 \mathrm{TeV}$. In PandaX-II, the typical momentum transfer is $10-50 \mathrm{MeV}$ for the $\mathrm{DM}$ mass $10 \mathrm{GeV}-1 \mathrm{TeV}$ with a heavy mediator. Thus, for $m_{\phi} \gtrsim 100 \mathrm{MeV}$, the observed limits quickly approach to the results from the recent WIMP searches in PandaX-II [22]. When the mediator decreases, the signal spectrum peaks towards low recoil energies, as shown in Fig. 1. Accordingly, the limits become weak, as the detection efficiency decreases significantly for $S 1$ below 10 PE. For comparison, we also show the results converted from Del Nobile et al. [6], where the authors reported limits on the mixing parameter in the context of an SIDM model (see below) by recasting an early LUX result. We see that our results improve significantly from previous ones. In the right panel, we show the limits vs the DM mass for $m_{\phi}=1 \mathrm{MeV}$, $10 \mathrm{MeV}$, and $1 \mathrm{GeV}$. For $m_{\phi}=1 \mathrm{GeV}$, the exclusion limits agree with our recent WIMP results [22], but become significantly weaker as $m_{\phi}$ decreases to $10 \mathrm{MeV}$ or less.

Our results have important implications in DM direct detection. For DM models with a light mediator $\left(m_{\phi} \lesssim q\right)$,

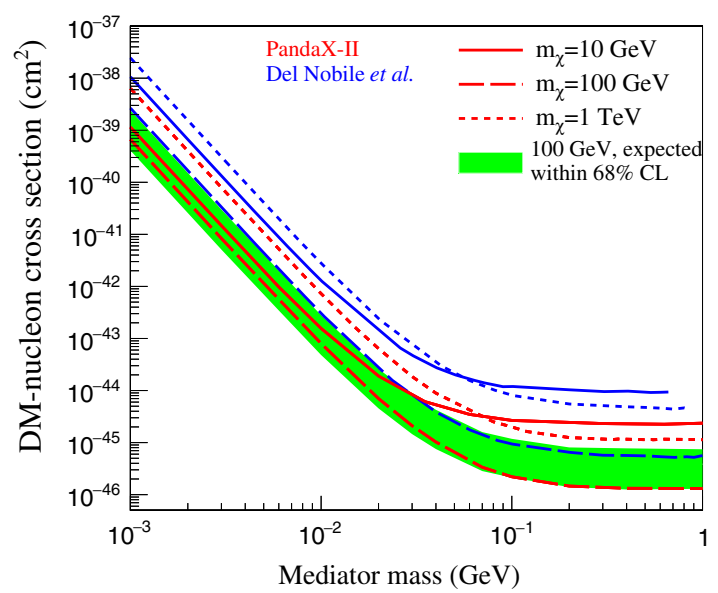

the contact-interaction approach can overestimate the direct detection sensitivity by orders of magnitude, and a full treatment of the scattering amplitude is required. From the model-building perspective, light-mediator DM models provide a natural mechanism to suppress the detection signal by populating events near the detector threshold limit. The analysis so far is based on the general form given in Eq. (1). In what follows, we apply our results to constrain the SIDM models [4] by explicitly calculating $\left.\sigma\right|_{q^{2}=0}$. In these models, the force carrier mediating DM self-interactions has a mass $\sim 1 \mathrm{MeV}-1 \mathrm{GeV}$.

Following Ref. [4], we assume DM is a Dirac fermion and it couples to a light mediator $\phi$. If $\phi$ is a vector (scalar) particle, it can couple to SM fermions through $\gamma / Z$ (Higgs) mixing. The DM-nucleon cross section in the limit of $q^{2}=0$ can be written as

$$
\left.\sigma\right|_{q^{2}=0}=\frac{16 \pi \alpha_{\mathrm{SM}} \alpha_{\chi} \mu_{p}^{2}}{m_{\phi}^{4}}\left(\frac{\epsilon_{p} Z+\epsilon_{n}(A-Z)}{A}\right)^{2},
$$

where $\alpha_{\mathrm{SM}}$ and $\alpha_{\chi}$ are the fine structure constants in the visible and dark sectors, respectively, $\epsilon_{p, n}$ are the effective proton or neutron couplings, and $Z$ is the proton number of the nucleus. For photon kinetic mixing or $Z$ mixing, $\epsilon_{p, n}$ are given by

$$
\epsilon_{p}=\epsilon_{\gamma}+\frac{\epsilon_{Z}}{4 s_{W} c_{W}}\left(1-4 s_{W}^{2}\right), \quad \epsilon_{n}=-\frac{\epsilon_{Z}}{4 s_{W} c_{W}},
$$

where $s_{W}$ and $c_{W}$ are the sine and cosine of the weak mixing angle, and $\epsilon_{\gamma, Z}$ is the photon kinetic or $Z$ mixing parameter. For Higgs mixing, they are

$$
\epsilon_{p, n}=\frac{m_{p, n} \epsilon_{H}}{e V}\left(1-7 f_{T G}^{p, n} / 9\right),
$$

where $\epsilon_{H}$ is the Higgs mixing parameter, $e$ is the electron charge, $V$ is the vacuum expectation value of the Higgs

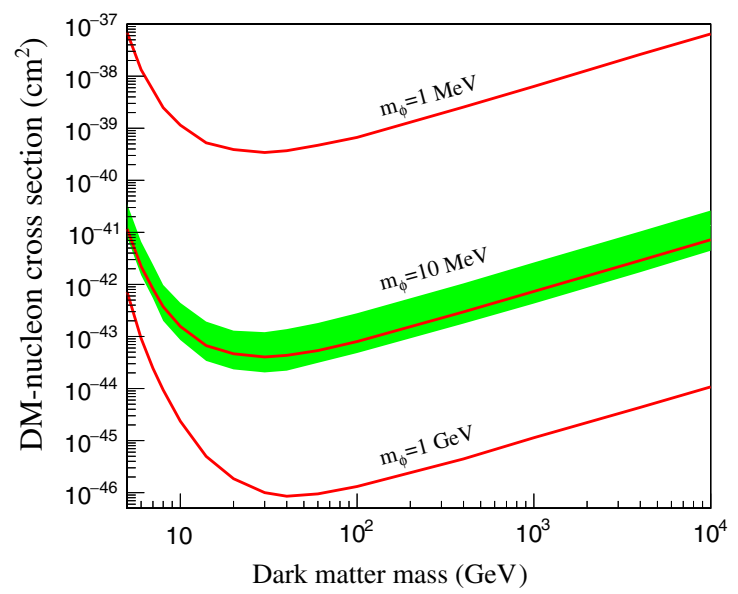

FIG. 2. PandaX-II 90\% C.L. upper limits (red) on the zero-momentum DM-nucleon cross section for DM models with a light mediator. Left: the cross section limits vs the mediator mass for three representative DM masses $10 \mathrm{GeV}, 100 \mathrm{GeV}$, and $1 \mathrm{TeV}$. The limits converted from previous results from Ref. [6] are included for comparison (blue). Right: the limits vs DM mass for mediator masses $1 \mathrm{MeV}, 10 \mathrm{MeV}$, and $1 \mathrm{GeV}$. The green bands denote the $\pm 1 \sigma$ sensitivity for the given model parameters. 
field, and $f_{T G}^{p, n}$ is determined by the gluon hadronic matrix element, which we take $f_{T G}^{p, n}=0.943$ [26].

We consider four cases. One is asymmetric SIDM with photon kinetic mixing. Asymmetric SIDM arises from the possibility that DM and anti-DM particles are not equally populated in the early Universe due to a primordial DM-number asymmetry [27,28]. Other three are symmetric SIDM with photon kinetic mixing, $Z$ mixing or Higgs mixing. For asymmetric SIDM, we set $\alpha_{\chi}$ to be 0.01, a choice motivated by the value of the electromagnetic fine structure constant in the SM [6]. For symmetric SIDM, the DM relic density is set by the annihilation process $\chi \bar{\chi} \rightarrow \phi \phi$, which sets $\alpha_{\chi}$ values as Ref. [4], $\alpha_{\chi} \approx$ $4 \times 10^{-5} \times\left(m_{\chi} / \mathrm{GeV}\right)$ for photon kinetic mixing or $Z$ mixing, and $\alpha_{\chi} \approx 10^{-4} \times\left(m_{\chi} / \mathrm{GeV}\right)$ for Higgs mixing.

For each case, astrophysical observations set a preferred region in the $m_{\phi}-m_{\chi}$ plane, where the self-scattering cross section per mass in dwarf galaxies is $\sim 0.1-10 \mathrm{~cm}^{2} / \mathrm{g}[4,6]$.
On the other hand, for a given DM mass, direct detection experiments put a constraint on the combination of the mixing parameter and the mediator mass. To present our limits in the $m_{\phi}-m_{\chi}$ plane, we will assume certain values of the mixing parameter. Note that Kaplinghat et al. [4] have reinterpreted an early XENON100 WIMP search result [29] to constrain the four cases, where a constant momentum transfer was assumed in calculating the total signal event. Furthermore, Del Nobile et al. [6] simulated full energy spectra and recasted results from early LUX [30] and SuperCDMS WIMP searches [31] to further constrain the asymmetric one. The present study uses the largest data set to date, and applies the complete analysis machinery in PandaX-II, including a thorough modeling of the detector response to signal and background based on the calibration data.

Figure 3 shows the $90 \%$ C.L. lower limits on the $\left(m_{\phi}\right.$, $m_{\chi}$ ) parameter region for four SIDM models. Our limits
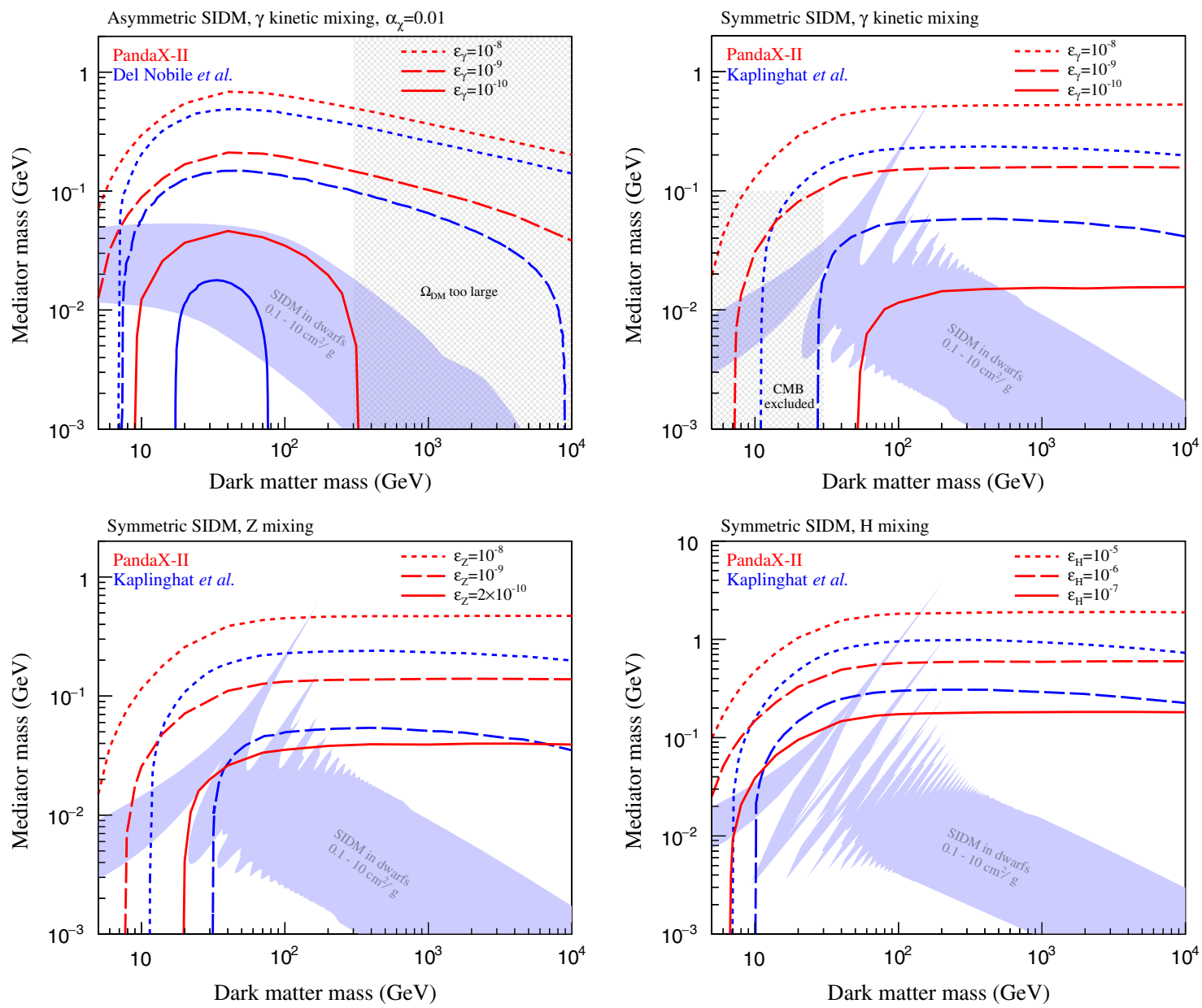

FIG. 3. PandaX-II $90 \%$ C.L. lower limits (red) on the $\left(m_{\phi}, m_{\chi}\right)$ plane for four SIDM models: asymmetric SIDM with photon kinetic mixing (upper left), symmetric SIDM with photon kinetic mixing (upper right), symmetric SIDM with $Z$ mixing (lower left), and symmetric SIDM with Higgs mixing (lower right). The shaded regions are favored by the observations in dwarf galaxies, taken from Refs. [4,6]. Previous limits (blue) from Refs. [4,6] are also shown for comparison. Cross shaded (upper): the DM abundance is too large for $m_{\chi} \gtrsim 300 \mathrm{GeV}$ (left); the region for $m_{\chi} \gtrsim 30 \mathrm{GeV}$ is excluded by the CMB observations (right), taken from Ref. [4]. 
(red) are reported at three $\epsilon_{\gamma}$ values, $10^{-8}, 10^{-9}$, and $10^{-10}$. Previous limits from Del Nobile et al. [6] by recasting a LUX result are included for comparison. The SuperCDMS limits are significantly weaker and not shown here. The shaded region is favored by observations in dwarf galaxies. Overall, a heavier DM particle requires a lighter mediator to enhance the self-scattering cross section, while keeping $\sigma_{\chi \chi} / m_{\chi}$ constant. If $\epsilon_{\gamma}>10^{-9}$, our results exclude all favored regions with $m_{\chi} \gtrsim 7 \mathrm{GeV}$. Even for $\epsilon_{\gamma}=10^{-10}$, we exclude a significant part of the favored region for DM masses ranging from 10 to $300 \mathrm{GeV}$. Previous limits are significantly weaker, in particular for a small mixing parameter.

The remaining panels in Fig. 3 are for symmetric SIDM models, with photon kinetic mixing, $Z$ mixing or Higgs mixing. Our limits are significantly stronger than the previous ones [4]. The features in the shaded SIDM region are due to the quantum resonant effect of attractive DM self-interactions [32]. For photon kinetic mixing $\epsilon_{\gamma}>10^{-9}$, our results exclude most of favored region by observations in dwarf galaxies for $m_{\chi} \geq 7 \mathrm{GeV}$. Even for $\epsilon_{\gamma}>10^{-10}$, we exclude a large parameter space. Similarly, almost all the favored heavy DM region is excluded for $Z$ mixing and $\epsilon_{Z}>10^{-9}$. Our results are not yet sensitive to $\epsilon_{Z}=10^{-10}$, but for $\epsilon_{Z}=2 \times 10^{-10}$, we can exclude a large portion of the favored region. For Higgs mixing, almost all the favored region shown is excluded if $\epsilon_{H}>10^{-6}$. We see that in most of the parameter region favored by the astrophysical observations, our results are sensitive to the mixing parameter as small as $\sim 10^{-10}$ for photon kinetic or $Z$ mixing, and $10^{-7}$ for Higgs mixing. The latter is weaker due to the suppression factor of $m_{p, n} / V$ in Eq. (5).

In conclusion, using a combined data corresponding to a total exposure of 54 ton day from the PandaX-II experiment, we have presented upper limits on the DM-nucleon scattering cross section with the mediator mass ranging from $1 \mathrm{MeV}$ to $1 \mathrm{GeV}$. The mediator mass plays a critical role in setting the exclusion limits for these models and a full analysis of the scattering amplitude is required. We further interpreted them to constrain the parameter space in the context of the SIDM models with a light mediator mixing with SM particles, complementing constraints from astrophysical observations. These are the first kind of results reported by a direct detection experimental collaboration. With more data from PandaX, particularly the future multi-ton scale experiment at CJPL, we will continue to probe the DM interaction with a light mediator and the selfinteracting nature of DM.

This project has been supported by a 985-III grant from Shanghai Jiao Tong University, grants from the National Science Foundation of China (No. 11435008, No. 11505112, No. 11525522, and No. 11755001), and a grant from the Ministry of Science and Technology of China (No. 2016YFA0400301). We thank the support of grants from the Office of Science and Technology, Shanghai Municipal Government (No. 11DZ2260700, No. 16DZ2260200, and No. 18JC1410200), and the support from the Key Laboratory for Particle Physics, Astrophysics and Cosmology, Ministry of Education. This work is supported in part by the Chinese Academy of Sciences Center for Excellence in Particle Physics (CCEPP) and Hongwen Foundation in Hong Kong. Finally, we thank the following organizations for indispensable logistics and other supports: the CJPL administration and the Yalong River Hydropower Development Company Ltd. HBY acknowledges support from U.S. Department of Energy under Grant No. de-sc0008541.

*Collaboration Spokesperson.

xdji@sjtu.edu.cn

Corresponding author.

yong.yang@sjtu.edu.cn

Corresponding author.

haiboyu@ucr.edu

[1] G. Bertone, D. Hooper, and J. Silk, Phys. Rep. 405, 279 (2005).

[2] R. Foot, Phys. Rev. D 69, 036001 (2004).

[3] N. Fornengo, P. Panci, and M. Regis, Phys. Rev. D 84, 115002 (2011).

[4] M. Kaplinghat, S. Tulin, and H.-B. Yu, Phys. Rev. D 89, 035009 (2014).

[5] T. Li, S. Miao, and Y.-F. Zhou, J. Cosmol. Astropart. Phys. 03 (2015) 032.

[6] E. Del Nobile, M. Kaplinghat, and H.-B. Yu, J. Cosmol. Astropart. Phys. 10 (2015) 055.

[7] F. Kahlhoefer, S. Kulkarni, and S. Wild, J. Cosmol. Astropart. Phys. 11 (2017) 016.

[8] C. Boehm and P. Fayet, Nucl. Phys. B683, 219 (2004).

[9] M. Pospelov, A. Ritz, and M. B. Voloshin, Phys. Lett. B 662, 53 (2008).

[10] J. L. Feng, H. Tu, and H.-B. Yu, J. Cosmol. Astropart. Phys. 10 (2008) 043.

[11] R. Foot and S. Vagnozzi, Phys. Rev. D 91, 023512 (2015).

[12] M. Blennow, S. Clementz, and J. Herrero-Garcia, J. Cosmol. Astropart. Phys. 03 (2017) 048.

[13] N. Arkani-Hamed, D. P. Finkbeiner, T. R. Slatyer, and N. Weiner, Phys. Rev. D 79, 015014 (2009).

[14] M. Pospelov and A. Ritz, Phys. Lett. B 671, 391 (2009).

[15] A. Kamada, M. Kaplinghat, A. B. Pace, and H.-B. Yu, Phys. Rev. Lett. 119, 111102 (2017).

[16] M. Kaplinghat, S. Tulin, and H.-B. Yu, Phys. Rev. Lett. 116, 041302 (2016).

[17] S. Tulin and H.-B. Yu, Phys. Rep. 730, 1 (2018).

[18] I. Baldes, M. Cirelli, P. Panci, K. Petraki, F. Sala, and M. Taoso, arXiv:1712.07489.

[19] M. Battaglieri et al., arXiv:1707.04591.

[20] A. Tan et al. (PandaX-II Collaboration), Phys. Rev. D 93, 122009 (2016).

[21] A. Tan et al. (PandaX-II Collaboration), Phys. Rev. Lett. 117, 121303 (2016). 
[22] X. Cui et al. (PandaX-II Collaboration), Phys. Rev. Lett. 119, 181302 (2017).

[23] C. Savage, G. Gelmini, P. Gondolo, and K. Freese, J. Cosmol. Astropart. Phys. 04 (2009) 010.

[24] G. Cowan, K. Cranmer, E. Gross, and O. Vitells, Eur. Phys. J. C 71, 1554 (2011); 73, 2501 (2013).

[25] E. Aprile et al. (XENON100 Collaboration), Phys. Rev. D 84, 052003 (2011).

[26] B. Bhattacherjee, S. Matsumoto, S. Mukhopadhyay, and M. M. Nojiri, J. High Energy Phys. 10 (2013) 032.

[27] K. M. Zurek, Phys. Rep. 537, 91 (2014).
[28] K. Petraki and R. R. Volkas, Int. J. Mod. Phys. A 28, 1330028 (2013).

[29] E. Aprile et al. (XENON100 Collaboration), Phys. Rev. Lett. 109, 181301 (2012).

[30] D. S. Akerib et al. (LUX Collaboration), Phys. Rev. Lett. 112, 091303 (2014).

[31] R. Agnese et al. (SuperCDMS Collaboration), Phys. Rev. Lett. 112, 241302 (2014).

[32] S. Tulin, H.-B. Yu, and K. M. Zurek, Phys. Rev. Lett. 110, 111301 (2013). 